\title{
Neglected infectious diseases in the geriatrics
}

\section{Abrar Abdulfattah Al Yamani ${ }^{1 *}$, Yahya Mohammed Falqi², Yussif Mohammed Alnawar', Lama Mohammed Almahrous ${ }^{4}$, Haitham Ahmed Alwael ${ }^{5}$, Ali Ibrahim Al Muhaif ${ }^{6}$, Hebah Adel Mansour ${ }^{7}$, Ghadeer Adil Banjar8, Tameem Abdullah Sabrah', Hanady Mohammed Idreis ${ }^{10}$, Yousef Shamlan Al Harban ${ }^{11}$}

\author{
${ }^{1}$ Obhur Primary Health Care Center, King Abdullah Medical Complex, Jeddah, Saudi Arabia \\ ${ }^{2}$ College of Medicine, Medical University of Silesia, Katowice, Poland \\ ${ }^{3}$ Primary Healthcare Center, Ministry of Health, Medina, Saudi Arabia \\ ${ }^{4}$ College of Medicine, National University of Ireland, Galway, Ireland \\ ${ }^{5}$ Public Health, King Fahad General Hospital, Jeddah, Saudi Arabia \\ ${ }^{6}$ College of Medicine, Medical University of Lodz, Lodz, Poland \\ ${ }^{7}$ Department of Medicine, Jeddah Eye Hospital, Jeddah, Saudi Arabia \\ ${ }^{8}$ Primary Healthcare Center, King Fahad General Hospital, Jeddah, Saudi Arabia \\ ${ }^{9}$ College of Medicine, King Khalid University, Abha, Saudi Arabia \\ ${ }^{10}$ Employee Clinic, King Fahad General Hospital, Jeddah, Saudi Arabia \\ ${ }^{11}$ College of Medicine, Royal College of Surgeons in Ireland Medical University of Bahrain, Muharraq, Kingdom of \\ Bahrain
}

Received: 24 November 2021

Revised: 13 December 2021

Accepted: 14 December 2021

\section{*Correspondence:}

Dr. Abrar Abdulfattah Al Yamani,

E-mail: abrar-yamani@gmail.com

Copyright: (C) the author(s), publisher and licensee Medip Academy. This is an open-access article distributed under the terms of the Creative Commons Attribution Non-Commercial License, which permits unrestricted non-commercial use, distribution, and reproduction in any medium, provided the original work is properly cited.

\begin{abstract}
Infectious diseases in the elderly population pose a significant threat to their lives. Neglected tropical diseases significantly impact the health of the affected patients and populations at risk. Reports show that many of these disorders are among the highest ten most typical causes of disability-adjusted life years. In the present literature review, we have discussed the most common neglected tropical infections in geriatrics based on data from the current studies in the literature. Different infections can affect the geriatric population. However, evidence shows that this population is susceptible to developing severe disease-related conditions. This has been reported with dengue infection, onchocerciasis, and cholera. It has been demonstrated that ocular lesions and other clinical manifestations are highest among the elderly population with onchocerciasis. Severe dengue and dengue hemorrhagic fever are also reported at a high rate in this age group. Concurrent infections and disorders were documented with many of these infections, probably due to reduced immunity. Socioeconomic factors, co-morbidities, access to healthcare settings, environmental factors, sanitation, clustering, and overcrowding contribute to the frequency of neglected tropical diseases in the elderly. Further studies are still needed because the current report is scarce, which might underestimate the current evidence.
\end{abstract}

Keywords: Geriatrics, Infection, Neglected clinical characteristics

\section{INTRODUCTION}

Neglected tropical diseases are a particular category under infectious diseases. This category includes different subsets of disorders, owing to different parasitological and microbiological species. ${ }^{1}$ The mechanism of infection of most of these diseases is poorly understood. Besides, the clinical presentation, diagnosis, and management are remarkably different among the relevant investigations. Nevertheless, evidence shows that these conditions are 
highly prevalent in the poorest communities globally, being responsible for significant morbidities and high rates of deaths. ${ }^{2,3}$

Neglected tropical diseases significantly impact the health of the affected patients and populations at risk. ${ }^{4}$ Geriatric patients are at increased risk of developing these events due to several factors, including poor health status, susceptibility to infections, impaired immunity, and decreased access to healthcare settings. Some neglected infections were reported, most specifically among elderly patients. ${ }^{5,6}$ These reports are scarce. Therefore, the impact of these reports might be underutilized. It is essential to conduct comparative investigations to highlight the burden of these conditions. Accordingly, we aim to conduct the current study to discuss the most common neglected tropical infections in the geriatric population, based on reports in the literature.

\section{METHODS}

This literature review is based on an extensive literature search in Medline, Cochrane, and EMBASE databases which was performed on $27^{\text {th }}$ November 2021 using the medical subject headings (MeSH) or a combination of all possible related terms, according to the database. To avoid missing potential studies, a further manual search for papers was done through Google Scholar while the reference lists of the initially included papers. Papers discussing neglected infectious diseases in the geriatrics were screened for useful information. No limitations were posed on date, language, age of participants, or publication type.

\section{DISCUSSION}

Different tropical diseases were reported in the literature. These include trachoma, soil-transmitted helminths, schistosomiasis, onchocerciases, leprosy, leishmaniasis, African human trypanosomiasis, and lymphatic filariasis. Other tropical infections include dracunculiasis, dengue fever, cholera, Chagas disease, and Buruli ulcer. Unfortunately, not many studies in the literature were published to elaborate on the different aspects of neglected tropical diseases in the elderly. Nevertheless, evidence shows that many of the reported infections were significantly associated with disability-adjusted life years, among the ten most common causes of these events. ${ }^{7-13}$ However, it should be noted that estimates of these events are not remarkably accurate, and some diseases are underestimated. Furthermore, it has been furtherly demonstrated that most of the reported neglected tropical diseases are focally transmitted. Accordingly, it has been shown that these events' clinical presentations and epidemiological characteristics are significant variants across the different populations. Accordingly, this might indicate that applying practical interventional approaches can significantly enhance the outcomes and eliminate the high prevalence and clusters in the vulnerable groups. Moreover, it is essential to identify the risk factors predisposed to these disorders' development and high prevalence to apply more adequate interventions.

Some studies reported that dengue is more severe in the elderly population as compared to the younger ones. For example, a previous investigation conducted in Singapore by Rowe et al. ${ }^{14}$ included old-age participants $(\geq 60$ years old) with dengue fever. The authors demonstrated that higher rates of urinary tract infections and pneumonia were noticed among the elderly. This might be attributed to the increased frequency of inserting different medical devices, like urinary catheters and indwelling, in addition to the increased duration of hospital stay. Moreover, this population group also had higher rates of hospital admissions. In the same context, it has been demonstrated that severe dengue and dengue hemorrhagic fever was most frequently reported in the elderly population. However, it should be noted that the authors indicated that there was no association between these worsened clinical outcomes and increased mortality. ${ }^{14}$ Previous investigations also indicated the development of similar outcomes in the same population. ${ }^{15,16}$ Furthermore, other previous investigations indicated that mortality was significantly correlated with the disease. ${ }^{15,17,18}$

Severe bleeding and severe plasma leakage were the most common manifestations reported in the elderly with severe dengue events. However, related weaning signs were less common among this study group. In this context, a previous study by Thein et al demonstrated that severe dengue or dengue hemorrhagic fever was not associated with any of the reported clinical outcomes and complications. ${ }^{19}$ On the other hand, specific characteristics included clinical accumulation of fluids, rapid drop in platelet volume associated with hematocrit rise, persistent vomiting, and hepatomegaly. ${ }^{19}$ In addition, plasma leakage has been reported to be a characteristic of dengue hemorrhagic fever, while bleeding is more frequently reported in cases with secondary dengue cases. ${ }^{20}$ In this context, it has been demonstrated that past infections have been higher among the elderly than younger patients. ${ }^{21} \mathrm{~A}$ previous epidemiological study reported that a history of dengue was prevalent in $88.9 \%$ in the included elderly population (55-74 years old) compared to $17.2 \%$ in young adults (18-24 years old). ${ }^{21}$

The risk of dengue hemorrhagic fever has been previously associated with having a history of previous dengue infection. ${ }^{14,17}$ The presence of co-morbidities might also attribute to the development of severe dengue in this vulnerable population. For instance, diabetes and hypertension were reported to be potential risk factors for developing severe dengue and dengue hemorrhagic fever. ${ }^{14,22}$ This was further indicated in a previous study that compared these outcomes with patients without comorbidities. $^{22}$ Diabetes mellitus can significantly impair the immune functions in the affected patients, and therefore, the association might be understood with developing dengue hemorrhagic fever. ${ }^{23,24}$ On the other 
hand, there is no clear evidence supporting the association between hypertension and severe dengue.

Evidence also indicates that different infections are concomitantly reported among elderly patients with dengue infection. For instance, previous studies reported that Staphylococcus aureus, leptospirosis, and malaria are common infections reported in this population group. ${ }^{25-27}$ The presence of co-infections has been attributed to the fact that dengue can significantly modulate the immune systems of the affected patients by impairing T-cell proliferation. $^{28}$ In the same context, evidence furtherly shows that impaired cytokine response and defects in B and $\mathrm{T}$ cell functions are significant features of the elderly population. ${ }^{23}$ Leo et al reported that bacteremia was present in $14.3 \%$ of the cases that died from dengue. ${ }^{29}$ In the same context, another study in Taiwan reported that acute renal failure and prolonged fever (more than five days) were significant predictors of bacteremia in patients with dengue hemorrhagic fever. The authors furtherly demonstrated that concurrent infections were higher among patients with leukocytosis. However, the association was not significant. ${ }^{30}$ Another study showed that length of hospital stay and nosocomial infections were higher among elderly patients with dengue with neutrophilia. ${ }^{14}$ Therefore, these clinical manifestations and potential complications should be clinically considered for these patients to enhance the diagnosis and management for the elderly and improve the outcomes. ${ }^{23,31}$

A previous study also emphasized the impact of aging on schistosomiasis infections. Based on previous reports from Brazil, the authors demonstrated that the prevalence of schistosomiasis among patients over 60 years of age is high and should no longer be ignored. Furthermore, impairing T-cell functions has been reported to be the primary mechanism by which aging is significantly associated with chronic schistosomiasis. ${ }^{32}$ Some reports were also previously published regarding helminths infections, indicating the prevalence of associated blindness with onchocerciasis in the elderly population.

Nwosa conducted a cross-sectional survey in Nigeria and included elderly patients $>50$ years of age in this context. The authors reported that the prevalence of visual impairment, monocular and bilateral blindness, were $23.7 \%, 11.9 \%$, and $8.6 \%$, respectively. Cataract was the most commonly reported reason for visual impairment in the studied group. Moreover, it has been demonstrated that other causes of blindness include refractive errors, onchocerciasis, glaucoma, and age-related macular degeneration. These findings indicate the association between onchocerciasis and visual impairment in the elderly population. ${ }^{33}$ Many other investigations in the literature also reported various clinical characteristics and ocular manifestations among elderly patients with onchocerciasis. Schwart et al reported that the prevalence of blindness among the elderly population with onchocerciasis was $77.6 \% .{ }^{34}$ Akogun estimated that only $29.8 \%$ of the elderly population were blind, $27.4 \%$ had punctate opacity, and $16.6 \%$ had impaired vision. ${ }^{35}$ Another case report by Sufi and Tukur also demonstrated that onchocerciasis among the elderly population resulted in 2 cases of blindness. ${ }^{36}$ Okoro et al also reported that the prevalence of blindness secondary to onchocerciasis was $28.5 \% .^{37}$

A case series by Dozie et al reported six elderly patients with blindness secondary to onchocerciasis. ${ }^{38}$ Most of the included studies in the literature included participants $\geq 50$ years of age. The reported rates for ocular complications are hugely variable across the different investigations in the literature, ranging between $18.2 \%$ to $100 \% .{ }^{39}$ Other manifestations of onchocerciasis in the elderly population were also previously reported in the literature and extensively discussed. ${ }^{39}$ Evidence shows that infection to the elderly population by onchocerca volvulus significantly leads to impaired host immunity. Therefore, these patients are more susceptible to other conditions and infectious diseases. It has been demonstrated that these events will cumulatively decrease the life expectancy of the affected patients. Other studies also showed that other conditions as epilepsy, HIV infections, and glaucoma are more prevalent among the elderly population affected by onchocerciasis. ${ }^{40-43}$ These findings indicate the significant impact of onchocerciasis on the immunity of the affected patients, which remarkably contributes to the development of other comorbid conditions and further deterioration of the cases of the affected geriatric patients. ${ }^{39}$

Reports of cholera infections were also documented in the literature. It has been evidenced that severe complications are more frequent among the elderly population. Evidence shows the association between aging and cholera-induced severe hypotension-related events, like stroke, vomitinginduced aspiration pneumonia, and renal compromise. ${ }^{44}$ Other reports also indicated that severe cholera and associated high mortality rates are observed among adult populations. On the other hand, it has been demonstrated that studies conducted in endemic areas demonstrated that such infections more frequently impact younger patients. ${ }^{45}$

Reports based on recent outbreaks of the disease in Yemen indicated that around one-third of the elderly population infected by cholera died, indicating the high virulence of the disease in this population. However, the high rate was attributed to different factors unique to the situation in Yemen. These include the reduced access to healthcare resources and poor quality of care across the country since the war started. Moreover, malnutrition was also prevalent across the country due to the poor resources, contributing to a generalized state of impaired immunity. The high virulence of cholera also strengthens these factors due to the general poor sanitation settings across the country. ${ }^{46,47}$ Different risk factors were reported in the literature for neglected tropical diseases. Some of these factors will be discussed in the following section. For instance, different studies have stressed the impact of socio-demographic factors on the development and high prevalence rates of these infections. The reported social factors include 
occupation, religion, social class, education, urbanization, and nutrition. In addition, age might also represent a common risk factor for the distribution of the different tropical diseases. ${ }^{48,49}$ For instance, it has been demonstrated that blindness secondary to trachoma and onchocerciasis are highly prevalent among the elderly. On the other hand, estimates show that African human trypanosomiasis is highly prevalent among the adult population. At the same time, soil-transmitted helminths, schistosomiasis, and Buruli ulcer are the most typical infections among children. Lymphatic filariasis was also reported to clinically manifest at an old age. However, evidence shows that the infection mainly occurs at a significantly younger age. Environmental factors, housing, and clustering, sanitation, and water were also reported as significant risk factors for the vulnerability of some groups for the development of neglected tropical diseases and infections. ${ }^{1,50}$

\section{CONCLUSION}

Infectious diseases in the elderly population pose a significant threat to their lives. Reports show that many of these disorders are among the highest ten most typical causes of disability-adjusted life years. Different infections can affect the geriatric population. However, evidence shows that this population is susceptible to developing severe disease-related conditions. This has been reported with dengue infection, onchocerciasis, and cholera. It has been demonstrated that ocular lesions and other clinical manifestations are highest among the elderly population with onchocerciasis. Severe dengue and dengue hemorrhagic fever are also reported at a high rate in this age group. Concurrent infections and disorders were documented with many of these infections, probably due to reduced immunity. Socioeconomic factors, the presence of co-morbidities, access to healthcare settings, environmental factors, sanitation, clustering, and overcrowding contribute to the frequency of neglected tropical diseases in the elderly.

\section{Funding: No funding sources}

Conflict of interest: None declared

Ethical approval: Not required

\section{REFERENCES}

1. Feasey N, Jones M, Mabey DC, Solomon AW. Neglected tropical diseases. $\mathrm{Br}$ Med Bull. 2010;93:179-200.

2. Collier P. The bottom billion: Why the poorest countries are failing and what can be done about it. USA: Oxford University Press; 2008.

3. Hotez PJ, Fenwick A, Savioli L, Molyneux DH. Rescuing the bottom billion through control of neglected tropical diseases. Lancet. 2009;373(9674):1570-5.

4. Molyneux DH, Malecela MN. Neglected tropical diseases and the millennium development goals: why the "other diseases" matter: reality versus rhetoric. Parasit Vectors. 2011;4:234.

5. Gruchy S. Introducing the United Nations Millennium Declaration. J Theol Southern Africa. 2001;110:57.

6. WHO. Neglected tropical diseases, hidden successes, emerging opportunities, 2021. Available at: https://apps.who.int/iris/handle/10665. Accessed on 15 October 2021.

7. Silva NR, Brooker S, Hotez PJ, Montresor A, Engels D, Savioli L. Soil-transmitted helminth infections: updating the global picture. Trends Parasitol. 2003;19(12):547-51.

8. Hotez PJ, Molyneux DH, Fenwick A, Kumaresan J, Sachs SE, Sachs JD, et al. Control of neglected tropical diseases. N Engl J Med. 2007;357(10):101827.

9. Brooker S, Rowlands M, Haller L, Savioli L, Bundy DA. Towards an atlas of human helminth infection in sub-Saharan Africa: the use of geographical information systems (GIS). Parasitol Today. 2000;16(7):303-7.

10. King CH, Dickman K, Tisch DJ. Reassessment of the cost of chronic helmintic infection: a meta-analysis of disability-related outcomes in endemic schistosomiasis. Lancet. 2005;365(9470):1561-9.

11. Engels D, Savioli L. Reconsidering the underestimated burden caused by neglected tropical diseases. Trends Parasitol. 2006;22(8):363-6.

12. Smith JL, Haddad D, Polack S, Esch EM, Hooper PJ, Mabey DC, et al. Mapping the global distribution of trachoma: why an updated atlas is needed. PLoS Negl Trop Dis. 2011;5(6):973.

13. Frick KD, Hanson CL, Jacobson GA. Global burden of trachoma and economics of the disease. Am J Trop Med Hyg. 2003;69(5):1-10.

14. Rowe EK, Leo YS, Wong JG, Thein TL, Gan VC, Lee LK, et al. Challenges in dengue fever in the elderly: atypical presentation and risk of severe dengue and hospital-acquired infection. PLoS Negl Trop Dis. 2014;8(4):2777.

15. Rivera EJ, Pérez JG. Dengue severity in the elderly in Puerto Rico. Rev Panam Salud Publica. 2003;13(6):362-8.

16. Rivera EJ, Pérez JG. Dengue severity in the elderly in Puerto Rico. Rev Panam Salud Publica. 2003;13(6):362-8.

17. Liu CC, Huang KJ, Huang MC. High case-fatality rate of adults with dengue hemorrhagic fever during an outbreak in non-endemic Taiwan: risk factors for dengue-infected elders. American J Infect Dis. 2008;4(1):10-7.

18. Lin CH, Schiøler KL, Jepsen MR, Ho CK, Li SH, Konradsen F. Dengue outbreaks in high-income area, Kaohsiung City, Taiwan, 2003-2009. Emerging infectious diseases. 2012;18(10):1603-11.

19. Thein TL, Gan VC, Lye DC, Yung CF, Leo YS. Utilities and limitations of the World Health Organization 2009 warning signs for adult dengue 
severity. PLoS neglected tropical diseases. 2013;7(1):2023.

20. Rothman AL, Ennis FA. Immunopathogenesis of Dengue hemorrhagic fever. Virology. 1999;257(1):16.

21. Yew YW, Ye T, Ang LW. Seroepidemiology of dengue virus infection among adults in Singapore. Annals of the Academy of Medicine, Singapore. 2009;38(8):667-75.

22. Pang J, Salim A, Lee VJ. Diabetes with hypertension as risk factors for adult dengue hemorrhagic fever in a predominantly dengue serotype 2 epidemic: a case control study. PLoS neglected tropical diseases. 2012;6(5):1641.

23. Opal SM, Girard TD, Ely EW. The immunopathogenesis of sepsis in elderly patients. Clinical infectious diseases: an official publication of the Infectious Diseases Society of America. 2005;41(7):504-12.

24. Geerlings SE, Hoepelman AI. Immune dysfunction in patients with diabetes mellitus (DM). FEMS Immunol Med Microbiol. 1999;26(3-4):259-65.

25. Magalhães BM, Alexandre MA, Siqueira AM. Clinical profile of concurrent dengue fever and Plasmodium vivax malaria in the Brazilian Amazon: case series of 11 hospitalized patients. American J Tropical Med Hygiene. 2012;87(6):1119-24.

26. Sharp TM, Bracero J, Rivera A. Fatal human coinfection with Leptospira spp. and dengue virus, Puerto Rico, 2010. Emerg Infect Dis. 2012;18(5):878-80.

27. Chai LY, Lim PL, Lee CC. Cluster of Staphylococcus aureus and dengue co-infection in Singapore. Annals Academy Med. 2007;36(10):847-50.

28. Mathew A, Kurane I, Green S, Vaughn DW, Kalayanarooj S, Suntayakorn S, et al. Impaired T cell proliferation in acute dengue infection. J Immunol. 1999;162(9):5609-15.

29. Leo YS, Thein TL, Fisher DA, Low JG, Oh HM, Narayanan RL, et al. Confirmed adult dengue deaths in Singapore: 5-year multi-center retrospective study. BMC Infect Dis. 2011;11:123.

30. Lee IK, Liu JW, Yang KD. Clinical characteristics and risk factors for concurrent bacteremia in adults with dengue hemorrhagic fever. American J Trop Med Hyg. 2005;72(2):221-6.

31. Gaudio AR, Rinaldi S, Chelazzi C, Borracci T. Pathophysiology of sepsis in the elderly: clinical impact and therapeutic considerations. Curr Drug Targets. 2009;10(1):60-70.

32. Comin F, Speziali E, Oliveira R, Faria AM. Aging and immune response in chronic human schistosomiasis. Acta Trop. 2008;108(2-3):124-30.

33. Nwosu SN. Low vision in persons aged 50 and above in the onchocercal endemic communities of Anambra State, Nigeria. West Afr J Med. 2000;19(3):216-9.

34. Schwartz EC, Huss R, Hopkins A, Dadjim B, Madjitoloum P, Hénault C, et al. Blindness and visual impairment in a region endemic for onchocerciasis in the Central African Republic. Br J Ophthalmol. 1997;81(6):443-7.

35. Akogun OB. Eye lesions, blindness and visual impairment in the Taraba river valley, Nigeria and their relation to onchocercal microfilariae in skin. Acta Tropica. 1992;51(2):143-9.

36. Schwartz EC, Huss R, Hopkins A, Dadjim B, Madjitoloum P, Hénault C, et al. Blindness and visual impairment in a region endemic for onchocerciasis in the Central African Republic. Br J Ophthalmol. 1997;81(6):443-7.

37. Okoro N, Nwali U, Oli A, Innocent O, Somadina O, Shedrack E. The Prevalence and Distribution of Human Onchocerciasis in Two Senatorial Districts in Ebonyi State, Nigeria. American J Infect Dis Microbiol. 2014;2:39-44.

38. Dozie I, Onwuliri C, Nwoke B. Onchocerciasis in Imo state, Nigeria (2): the prevalence, intensity and distribution in the upper Imo river basin. Int J Environ Health Res. 2004;14(5):359-69.

39. Shintouo CM, Shey RA, Mets T, Vanhamme L, Souopgui J, Ghogomu SM, et al. Onchocerciasis Fingerprints in the Geriatric Population: Does Host Immunity Play a Role? Trop Med Infect Dis. 2021;6(3):153.

40. Gopinath R, Ostrowski M, Justement SJ, Fauci AS, Nutman TB. Filarial infections increase susceptibility to human immunodeficiency virus infection in peripheral blood mononuclear cells in vitro. J Infect Dis. 2000;182(6):1804-8.

41. Colebunders R, Siewe FN, Hotterbeekx A. Onchocerciasis-associated epilepsy, an additional reason for strengthening onchocerciasis elimination programs. Trends Parasitol. 2018;34(3):208-16.

42. Fodjo JN, Mandro M, Mukendi D, Tepage F, Menon S, Nakato S, Nyisi F, et al. Onchocerciasis-associated epilepsy in the Democratic Republic of Congo: Clinical description and relationship with microfilarial density. PLoS Negl Trop Dis. 2019;13(7):7300.

43. Egbert PR, Jacobson DW, Fiadoyor S, Dadzie P, Ellingson KD. Onchocerciasis: a potential risk factor for glaucoma. British J Ophthalmol. 2005;89(7):7968.

44. Ryan ET, Dhar U, Khan WA, Salam MA, Faruque AS, Fuchs GJ, et al. Mortality, morbidity, and microbiology of endemic cholera among hospitalized patients in Dhaka, Bangladesh. Am J Trop Med Hyg. 2000;63(1-2):12-20.

45. Phelps M, Perner ML, Pitzer VE, Andreasen V, Jensen PKM, Simonsen L. Cholera Epidemics of the Past Offer New Insights Into an Old Enemy. J Infect Dis. 2017;217(4):641-9.

46. Bellizzi S, Pichierri G, Cegolon L, Napodano CMP, Maher OA. Coordination during Cholera Outbreak Response: Critical Insights from Yemen. Am J Trop Med Hyg. 2021;105(5):1155-6.

47. Qaserah AM, Al AMA, Serouri AA, Khader YS. Risk Factors of Cholera Transmission in Al Hudaydah, 
Yemen: Case-Control Study. JMIR Public Health Surv. 2021;7(7).

48. Son PT, Reda A, Viet DC. Exchange transfusion in the management of critical pertussis in young infants: a case series. Vox sanguinis. 2021;116(9):976-82.

49. Thieu H, Bach Dat B, Nam NH. Antibiotic resistance of Helicobacter pylori infection in a children's hospital in Vietnam: prevalence and associated factors. Minerva Medica. 2020;111(5):498-501.
50. Hansen J, Chaignat C. Neglected tropical diseases: Equity and social etermin. 2010:135-57.

Cite this article as: Yamani AAA, Falqi YM, Alnawar YM, Almahrous LM, Alwael HA, Muhaif AIA, et al. Neglected infectious diseases in the geriatrics. Int J Community Med Public Health 2022;9:353-8. 